\title{
Liver function tests in identifying patients with liver disease
}

This article was published in the following Dove Press journal: Clinical and Experimental Gastroenterology

\author{
Zohair Ahmed' \\ Umair Ahmed ${ }^{2}$ \\ Saqib Walayat ${ }^{2}$ \\ Jinma Ren ${ }^{3}$ \\ Daniel K Martin ${ }^{4}$ \\ Harsha Moole ${ }^{2}$ \\ Sean Koppe ${ }^{5}$ \\ Sherri Yong 6 \\ Sonu Dhillon ${ }^{4}$ \\ 'Department of Gastroenterology \\ and Hepatology, University of Illinois, \\ Chicago, IL, USA; ${ }^{2}$ Department \\ of Internal Medicine, University \\ of Illinois College of Medicine, \\ Peoria, IL, USA; ${ }^{3}$ Department of \\ Center for Outcomes Research, \\ University of Illinois College \\ of Medicine, Peoria, IL, USA; \\ ${ }^{4}$ Department of Gastroenterology \\ and Hepatology, University of Illinois \\ College of Medicine, Peoria, IL, \\ USA; ${ }^{5}$ Department of Hepatology, \\ University of Illinois at Chicago, \\ Chicago, IL, USA; ' ${ }^{\text {Department }}$ of \\ Pathology, University of Illinois \\ College of Medicine, Peoria, IL, USA
}

Correspondence: Zohair Ahmed Division of Gastroenterology and Hepatology, University of Illinois at Chicago, 840 South Wood Street, Suite 7I8E, Chicago 606I2, IL, USA

$\mathrm{Tel}+\mathrm{I} 6306999247$

$\mathrm{Fax}+\mathrm{I} 3129965103$

Email Zahmed23@uic.edu
Background and aims: Many patients with liver disease come to medical attention once they have advanced cirrhosis or acute decompensation. Most often, patients are screened for liver disease via liver function tests (LFTs). There is very limited published data evaluating laboratory values with biopsy-proven stages of hepatic fibrosis. We set out to evaluate whether any correlation exists between routine LFTs and stages of hepatic fibrosis.

Methods: A large retrospective observational study on 771 liver biopsies was conducted for evaluating the stage of fibrosis with AST, ALT, INR, BUN, creatinine, platelets, alkaline phosphatase, bilirubin, and albumin. Mean and 95\% confidence intervals were used to describe the distributions of serum markers in different fibrosis stages. Multivariable generalized linear models were used and a two-tailed $P$-value was calculated.

Results: ALT was not statistically significant for any stage, and AST was statistically significant for stage 3 and 4 fibrosis. INR was statistically significant only in stage 4 disease but remained near the upper limit of normal range. Albumin failed to show a clinically relevant association. Platelets remained within normal laboratory range for all stages. The remaining laboratory values failed to show statistical and clinical significance.

Conclusion: The health care burden from chronic liver disease (CLD) will likely continue to rise, unless clinicians are made aware that normal or near normal laboratory findings may be seen in asymptomatic patients. Earlier identification of asymptomatic patients will allow for treatment with new promising modalities and decrease morbidity and mortality from CLD. Our study shows that laboratory values correlate poorly with liver disease.

Keywords: chronic liver disease, hepatic fibrosis, serum markers, cirrhosis, NAFLD, HCV

\section{Introduction}

Chronic liver disease (CLD) is a major cause of morbidity and mortality and is quickly becoming an increasing burden on the health care system. Both CLD and cirrhosis are the fifth leading cause of death in the 45-61 age group and 12th leading cause overall., ${ }^{1,2}$ In 2010, the National Center for Health Statistics (NCHS) and Centers for Disease Control and Prevention (CDC) reported 31,903 deaths from CLD and cirrhosis. This number is expected to increase steadily well in the next decade. The prevalence of cirrhosis in the general population is difficult to determine and was estimated to be 1 in $679(0.15 \%)$ in a recent study published by the National Institute of Health $(\mathrm{NIH}){ }^{3}$ This could represent a gross underestimation of 1 in $370(0.27 \%)$ as suggested in a recent study by Scagilone et al, where the prevalence was estimated based on the 2010 US census. Moreover in that study, $70 \%$ of the patients found to have cirrhosis were 
unaware of their disease. ${ }^{4}$ Due to the large functional reserve in the liver, most patients with CLD go undiagnosed until late in the course of the disease. Patients seek medical attention when the liver can no longer maintain homeostasis and begins decompensating with the onset of ascites, variceal bleeding, spontaneous bacterial peritonitis, encephalopathy, or jaundice.

Regardless of the etiology of the liver disease, these individuals undergo chronic persistent inflammation and progressive fibrosis, which leads to cirrhosis. ${ }^{5}$ This sustained liver injury leads to hepatocellular injury and excessive deposition of collagen by activated hepatic stellate cells (HSCs). HSCs play the most pivotal role in liver fibrogenesis and are the target of interest for future therapeutic modalities to prevent or reverse advanced hepatic fibrosis. Once activated HSCs release chemokines, the affected regions of liver parenchyma undergo unregulated deposition of the extracellular matrix resulting in the development of fibrosis. ${ }^{6-10}$ The extent and severity of the fibrosis on a liver biopsy determines the stage of the disease. Liver biopsy has been the gold standard for evaluating liver injury and staging the disease. Although there are several proposed schemes to stage fibrosis, the Batts and Ludwig system is most commonly used by pathologists in the USA. This model assesses the extent and location of the fibrosis, accompanying changes in parenchymal architecture and is expressed on a linear numerical scale of stage 0 (no fibrosis) to stage 4 (cirrhosis). ${ }^{11}$ Regardless of the etiology, prolonged inflammation leads to chronic liver injury, fibrosis, and often cirrhosis. Hepatic dysfunction and portal hypertension complicate cirrhosis as the liver decompensates. ${ }^{12}$ In the USA, hepatitis $\mathrm{C}$, hepatitis $\mathrm{B}$, alcoholic liver disease, and non-alcoholic fatty liver disease (NAFLD) are the important causes of chronic liver injury and progressive fibrosis.

Recently, there has been extensive interest in finding reliable non-invasive methods to diagnose the stage of liver disease via serological (APRI, FIB-4, Forns index, and FibroTest) and radiological (ultrasound and MRI elastography) tests. ${ }^{13-18}$ Most clinicians associate both acute and CLD with abnormal liver function tests (LFTs). However, there has been very little published data comparing the changes in laboratory values and stages of fibrosis. Identifying individuals at risk for chronic liver injury requires a high index of suspicion and a thorough history and physical examination. Laboratory studies such as LFTs identify some but not all patients with significant liver injury. In order to determine whether commonly used laboratory tests are valuable to identify patients with chronic liver injury, we conducted a retrospective study on 771 liver biopsies done at our institution and evaluated the laboratory values for each stage of fibrosis and cirrhosis. Our goal was to identify any common laboratory value or pattern that would help identify the individuals with advanced liver disease.

\section{Methods Design and population}

A retrospective observational study was conducted evaluating all liver biopsies at OSF Saint Francis Medical Center in Peoria, IL, USA, from 2010 to 2014. Liver biopsies done for metastatic liver disease, hepatic neoplasms (benign and malignant), biliary atresia, and fulminant liver failure were excluded, and a total of 771 liver biopsies were evaluated.

\section{Measures}

Batts and Ludwig scoring system was used to evaluate fibrosis stage: stage 0, no fibrosis; stage 1, portal fibrosis; stage 2, periportal fibrosis (including rare portal-portal septa); stage 3, septal fibrosis (with architectural distortion); and stage 4, cirrhosis. Serum markers such as AST, ALT, INR, BUN, creatinine $(\mathrm{Cr})$, platelets (Plts), alkaline phosphatase, total bilirubin, and albumin were studied within 90 days before and after liver biopsy.

\section{Ethical issue}

Written informed consent was obtained from all the study subjects for liver biopsy. This study was approved by the Institutional Review Board (IRB) at the University of Illinois College of Medicine in Peoria. Patient consent was not required to review their medical records by the IRB because the research involves no more than minimal risk to the subjects, and the waiver or alteration will not adversely affect the rights and welfare of the subjects. An adequate plan was developed to protect the identifiers from improper use and disclosure.

\section{Statistical analyses}

Mean and 95\% confidence intervals (CIs) were used to describe the distributions of serum markers in different fibrosis stages. Multivariable generalized linear models were used to examine the association between serum markers and fibrosis progress (stages $1-4$ vs stage 0 ). A two-tailed $P$-value was calculated for all tests and $P \leq 0.05$ was considered as statistically significant. All analyses were performed using SAS 9.4 (SAS Institute Inc., Cary, NC, USA).

\section{Results}

Among 771 patients with liver biopsies, the average age was $54.3( \pm 12.8)$ with a range of $20-91$ years old and there were equal numbers of males and females. The underlying liver 
diseases included: $253 \mathrm{HCV}$ patients (38.5\%), 159 NAFLD patients $(24.2 \%), 25$ alcoholic liver disease patients $(3.8 \%)$, $14 \mathrm{HBV}$ patients $(2.1 \%)$, and 206 patients with other diseases (31.4\%). Approximately one-third of the patients was alcoholic (33.7\%) but only 25 (3.8\%) had alcohol-related liver disease as a diagnosis at the time of liver biopsy. Approximately one-third of the patients (34.6\%) reported current use of tobacco products. The percentage of patients with fibrosis stages $0,1,2,3$, and 4 was $21.0 \%, 13.9 \%, 28.1 \%, 10.8 \%$ and $26.2 \%$, respectively (Table 1 ). The largest number of patients had periportal fibrosis (stage 2).

\section{AST}

The normal range of AST is 5-34 U/L. The AST values in the fibrosis stages 3 and 4 were significantly elevated when compared to stage 0 ( 70 vs $58, P<0.05 ; 73$ vs $58, P<0.01$, respectively). When stages 1 and 2 were compared to stage 0 , the difference was not significant ( 50 vs $58, P>0.05 ; 63$ vs $58, P>0.05$, respectively) (Table 2 , Figure 1 ).

\section{ALT}

The normal range of ALT is $0-55 \mathrm{U} / \mathrm{L}$. The ALT values in the fibrosis stages 2 and 3 were significantly elevated when compared to stage 0 ( 71 vs $56, P<0.05 ; 76$ vs $56, P<0.05$,

Table I Profile of patients

\begin{tabular}{lll}
\hline Items & Frequency & $\%$ \\
\hline Gender (male), $\mathrm{n}$ & 392 & 50.8 \\
Ethnicity, $\mathrm{n}$ & & \\
$\quad$ Caucasian & 626 & 81.2 \\
African American & 110 & 14.3 \\
Asian & 10 & 1.3 \\
Unknown & 3 & 0.4 \\
Others & 22 & 2.9 \\
Etiology, $\mathrm{n}$ & & \\
Hepatitis C & 253 & 38.5 \\
Alcoholic liver disease & 25 & 3.8 \\
Non-alcoholic fatty liver disease & 159 & 24.2 \\
Hepatitis B & 14 & 2.1 \\
Others & 206 & 31.4 \\
Alcohol use (yes), $\mathrm{n}$ & 260 & 33.7 \\
Tobacco use, $\mathrm{n}$ & & \\
Yes, currently use & 267 & 34.6 \\
Already quit & 258 & 33.5 \\
Never use & 246 & 31.9 \\
Fibrosis stage, $\mathrm{n}$ & & \\
0 & 162 & 21.0 \\
I & 107 & 13.9 \\
2 & 217 & 28.1 \\
3 & 83 & 10.8 \\
4 & 202 & 26.2 \\
Age (years), mean \pm SD & $54.3 \pm 12.8$ & \\
\hline
\end{tabular}

Abbreviation: SD, standard deviation. respectively). When stages 1 and 4 were compared to stage 0 , the $P$-value was not significant (64 vs $56, P>0.05$; 58 vs $56, P>0.05$, respectively) (Table 2 , Figure 1 ).

\section{Alkaline phosphatase}

The normal range of alkaline phosphatase is $50-125 \mathrm{U} / \mathrm{L}$. The alkaline phosphatase values in the fibrosis stages 1 and 4 were significantly lower when compared to stage 0 (120 vs $205, P<0.05 ; 152$ vs $205, P<0.05$, respectively). When stages 2 and 3 were compared to stage 0 , the $P$-value was not significant (167 vs $205, P>0.05 ; 158$ vs $205, P>0.05$, respectively) (Table 2, Figure 1).

\section{Total bilirubin}

The normal range of total bilirubin is $0.0-1.5 \mathrm{mg} / \mathrm{dL}$. The total bilirubin value in fibrosis stage 4 was significantly elevated when compared to stage $0(2.7$ vs $1.7, P<0.05)$. When stages 1,2 , and 3 were compared to stage 0 , the $P$-value was not significant $(0.8$ vs $1.7, P>0.05 ; 1.3$ vs $1.7, P>0.05 ; 2.0$ vs $1.7, P>0.05$, respectively) (Table 2 , Figure 2 ).

\section{INR}

The normal range of INR is $0.9-1.2$. The INR values in the fibrosis stages 2 and 4 were statistically significant when compared to stage 0 ( 1.07 vs $1.15, P<0.05 ; 1.27$ vs $1.15, P<0.05$, respectively). Yet, they failed to be clinically significant since they were fairly within normal limits. When stages 1 and 3 were compared to stage 0 , they were not clinically significant and the $P$-value was not statistically significant (1.08 vs 1.15 , $P>0.05 ; 1.11$ vs $1.15, P>0.05$, respectively) (Table 2, Figure 2).

\section{BUN}

The normal range of BUN is $8-26 \mathrm{mg} / \mathrm{dL}$. The BUN values in the fibrosis stage 4 was significantly lower when compared to stage 0 ( 16.8 vs $20.1, P<0.05)$. When stages 1,2 , and 3 were compared to stage 0 , the $P$-value was not significant (18.1 vs $20.1, P>0.05 ; 15.8$ vs $20.1, P>0.05 ; 16.5$ vs 20.1 , $P>0.05$, respectively) (Table 2 , Figure 1 ).

\section{$\mathrm{Cr}$}

The normal range of $\mathrm{Cr}$ is $0.6-1.0 \mathrm{mg} / \mathrm{dL}$ (female) and $0.7-1.3 \mathrm{mg} / \mathrm{dL}$ (male). The $\mathrm{Cr}$ values were not statistically significant in stages $1,2,3$, and 4 when compared to stage 0 (Table 2, Figure 2).

\section{Albumin}

The normal range of albumin is $3.5-5.0 \mathrm{~g} / \mathrm{dL}$. The albumin values in the fibrosis stages 1,2 , and 3 were statistically 
Table 2 Association between serum markers and fibrosis stage

\begin{tabular}{|c|c|c|c|c|c|c|c|}
\hline \multirow[t]{2}{*}{ Marker } & \multicolumn{3}{|c|}{ Adjusted model $^{a}$} & \multirow[t]{2}{*}{ Marker } & \multicolumn{3}{|c|}{ Adjusted model $^{\mathbf{a}}$} \\
\hline & Mean & SD & $P$-value & & Mean & SD & $P$-value \\
\hline \multicolumn{4}{|c|}{ AST (normal: 17-59) } & \multicolumn{4}{|c|}{ Total bilirubin (normal: $0.2-1.2$ ) } \\
\hline Stage 0 & 58.14 & 4.29 & Ref. & Stage 0 & I.7I & 1.03 & Ref. \\
\hline Stage I & 50.49 & 4.74 & 0.2283 & Stage I & 0.81 & 0.90 & 0.0872 \\
\hline Stage 2 & 63.39 & 4.27 & 0.2222 & Stage 2 & 1.33 & 0.89 & 0.4867 \\
\hline Stage 3 & 70.02 & 4.27 & 0.0423 & Stage 3 & 1.98 & 0.95 & 0.5085 \\
\hline Stage 4 & 73.25 & 4.28 & 0.0014 & Stage 4 & 2.73 & 0.92 & 0.0188 \\
\hline \multicolumn{4}{|c|}{ ALT (normal: 0-55) } & \multicolumn{4}{|c|}{ Albumin (normal: $3.5-5.0$ ) } \\
\hline Stage 0 & 55.65 & 10.46 & Ref. & Stage 0 & 3.48 & 0.21 & Ref. \\
\hline Stage I & 63.98 & 8.82 & 0.2226 & Stage I & 3.91 & 0.21 & 0.0007 \\
\hline Stage 2 & 70.58 & 8.26 & 0.0092 & Stage 2 & 3.81 & 0.18 & 0.0117 \\
\hline Stage 3 & 75.63 & 9.37 & 0.0025 & Stage 3 & 3.88 & 0.17 & 0.0031 \\
\hline Stage 4 & 58.24 & 9.43 & 0.7787 & Stage 4 & 3.34 & 0.18 & 0.0607 \\
\hline \multicolumn{4}{|c|}{ BUN (normal: 8-26) } & \multicolumn{4}{|c|}{$\mathrm{Cr}$ (normal: $0.7-1.3$ ) } \\
\hline Stage 0 & 20.10 & 5.14 & Ref. & Stage 0 & 1.00 & 0.28 & Ref. \\
\hline Stage I & 18.05 & 5.06 & 0.9556 & Stage I & 1.16 & 0.26 & 0.1355 \\
\hline Stage 2 & 15.75 & 4.78 & 0.2832 & Stage 2 & 1.01 & 0.27 & 0.4503 \\
\hline Stage 3 & 16.49 & 4.42 & 0.3162 & Stage 3 & 1.10 & 0.25 & 0.3717 \\
\hline Stage 4 & 16.80 & 4.46 & 0.0775 & Stage 4 & 1.07 & 0.24 & 0.6418 \\
\hline \multicolumn{4}{|c|}{ INR (normal: 0.9-I.2) } & \multicolumn{4}{|c|}{ Glucose (normal: 70-99) } \\
\hline Stage 0 & 1.15 & 0.06 & Ref. & Stage 0 & 111.47 & 7.12 & Ref. \\
\hline Stage I & 1.08 & 0.06 & 0.1258 & Stage I & 108.24 & 6.60 & 0.9646 \\
\hline Stage 2 & 1.07 & 0.06 & 0.0475 & Stage 2 & 119.28 & 5.69 & 0.0658 \\
\hline Stage 3 & 1.11 & 0.06 & 0.5516 & Stage 3 & 120.49 & 4.98 & 0.1373 \\
\hline Stage 4 & 1.27 & 0.06 & $<0.0001$ & Stage 4 & 117.95 & 5.67 & 0.2094 \\
\hline \multicolumn{4}{|c|}{ ALK (normal: 38-126) } & \multicolumn{4}{|c|}{ Platelets (normal: I40-440) } \\
\hline Stage 0 & 204.77 & 25.70 & Ref. & Stage 0 & 228.80 & 17.96 & Ref. \\
\hline Stage I & 119.54 & 24.02 & 0.0007 & Stage I & 225.76 & 19.32 & 0.3983 \\
\hline Stage 2 & 167.19 & 27.14 & 0.1173 & Stage 2 & 223.39 & 18.63 & 0.1514 \\
\hline Stage 3 & 157.84 & 26.82 & 0.0914 & Stage 3 & 195.95 & 17.49 & 0.0009 \\
\hline Stage 4 & 151.98 & 23.53 & 0.0070 & Stage 4 & 151.75 & 16.77 & $<0.000 \mid$ \\
\hline
\end{tabular}

Note: aMultivariable generalized linear model adjusted by age, gender, alcohol use and tobacco use.

Abbreviations: SD, standard deviation; Ref., reference; Cr, creatinine.

significant when compared to stage 0 ( 3.9 vs $3.5, P<0.05 ; 3.8$ vs $3.5, P<0.05 ; 3.9$ vs $3.5, P<0.05$, respectively). When stage 4 was compared to stage 0 , the $P$-value was not significant (3.3 vs $3.5, P>0.05$ ) (Table 2, Figure 2 ).

\section{Platelet}

The normal platelet $(\mathrm{Plt})$ range is $140-440(103 / \mu \mathrm{L})$. The Plt values in the fibrosis stages 3 and 4 were significantly lower when compared to stage 0 ( 196 vs $229, P<0.05 ; 152$ vs 229 , $P<0.05$, respectively). When stages 1 and 2 were compared to stage 0 , the $P$-value was not significant (226 vs $229, P>0.05$; 223 vs $229, P>0.05$, respectively) (Table 2 , Figure 1 ).

\section{Discussion}

CLD and cirrhosis have gained increasing attention due to the newly approved therapies for hepatitis $\mathrm{C}$ and the increasing obesity epidemic. Because of the morbidity and mortality associated with CLD and cirrhosis, there is significant utilization of health care resources. Identification of affected individuals is critical to help mitigate the progression of disease and, more importantly, identify those with end stage liver disease who are at significant risk for decompensation. Identification of those individuals with treatable disease, such as HCV, HBV, and autoimmune hepatitis, may slow or even reverse fibrosis and early cirrhosis. In the era of antiviral therapy, diet and lifestyle modification, and targeted molecular therapy trials in vivo, many may have the potential to experience some reversibility of their liver injury. ${ }^{19,20}$ Much research has centered on non-invasive means of rapidly identifying individuals with fibrosis such as serologic markers (APRI, FibroTest/FibroSure, Hepascore, and FibroSpect) and radiologic methods (ultrasound transient elastography and magnetic resonance elastography). However, before these novel methods are utilized, it is important for clinicians to recognize those at risk for liver disease. Although most clinicians rely on abnormal LFTs to identify such 


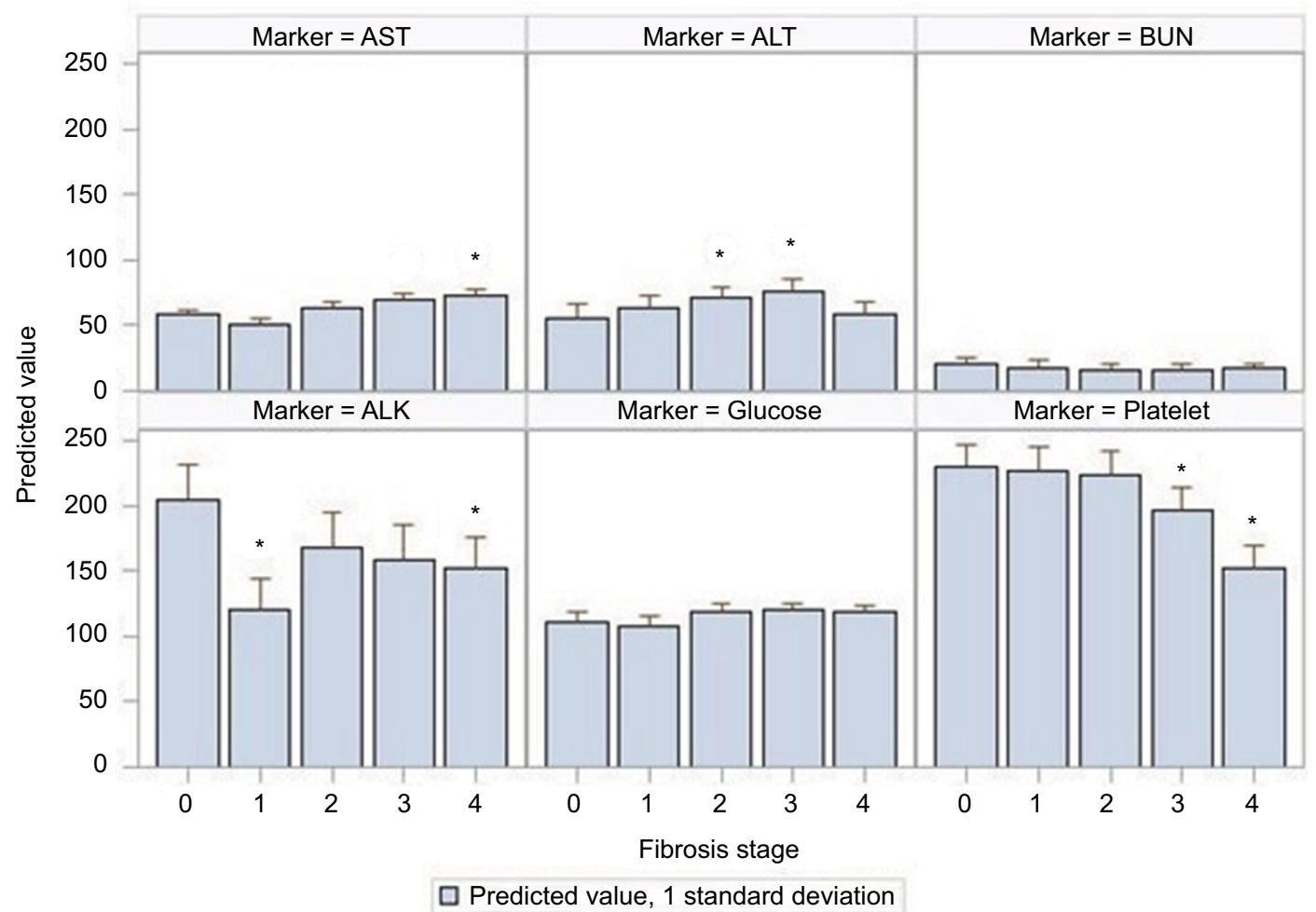

Figure I Six serum markers and fibrosis stage.

Notes: Multivariable generalized linear models were used to estimate the predicted values controlling for age, gender, alcohol use, and tobacco use. *Statistically significant difference $(P<0.05)$ compared to the fibrosis stage 0 .

Abbreviation: ALK, alkaline phosphatase.

individuals, many patients with CLD typically have normal to near normal LFTs.

Our retrospective study evaluated 771 liver biopsies and compared the Batts and Ludwig stage of fibrosis to serum markers such as AST, ALT, INR, BUN, Cr, Plt, alkaline phosphatase, total bilirubin, and albumin performed within 90 days of the biopsy.

INR and albumin are often regarded as important markers of synthetic function, and our study revealed a normal range of INR for all pre-cirrhotic stages. The INR was statistically significant for stage 4 fibrosis when compared to those individuals with no discernible liver disease; however, it remained near the upper limit of normal. Albumin is a major protein synthesized by the liver. We did not see a progressive decrease with advanced disease, and the albumin level in stage 4 remained close to the normal value and was not statistically significant. As such, makers for hepatic synthetic function (albumin and INR) do not appear to be a good measure of hepatic fibrosis.

In our study while there was a statistically significant decline in the Plt count for stage 3 and 4 diseases (Figure 1, Table 2), the absolute values remained in the normal range and thus Plt count itself is not a useful guide. Advanced liver disease results in a decrease in thrombopoietin production, and when cirrhosis develops, the resultant portal hypertension leads to splenic sequestration of Plts with subsequent decline in the Plt count.

AST and ALT are also the most common laboratory values that clinicians check when evaluating liver function. Based on our study, AST showed some progressive increase and was statistically significant in advanced liver disease, whereas ALT which is more liver specific did not show this correlation.

Our findings highlight the importance of clinicians to keep a high index of suspicion in the identification of patients with advanced liver disease. Often times, patients with advanced liver disease and cirrhosis have subtle changes in laboratory values, typically falling within the normal ranges. As such, routine laboratory studies should not be used as a sole screening tool to identify or exclude individuals suspected of having CLD. History and physical examination remain the cornerstone of the diagnostic workup, which unfortunately is often obtained after many patients present with acute variceal bleeding, jaundice, or ascites. The 


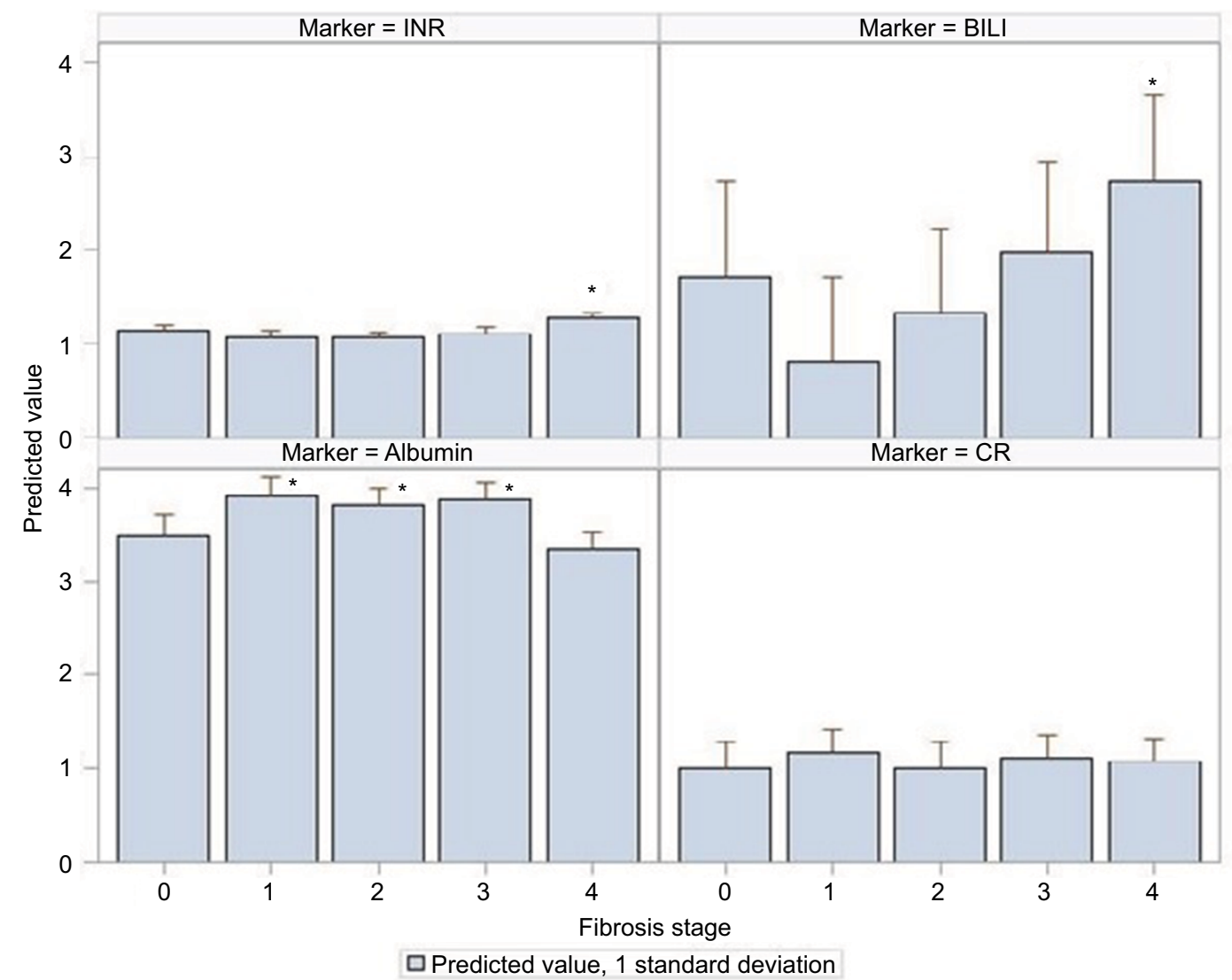

Figure 2 Four serum markers and fibrosis stage.

Notes: Multivariable generalized linear models were used to estimate the predicted values controlling for age, gender, alcohol use, and tobacco use. *Statistically significant difference $(P<0.05)$ compared to the fibrosis stage 0 .

Abbreviation: $\mathrm{Cr}$, creatinine.

health care burden from decompensated liver disease will likely continue to rise, unless clinicians are made aware that normal or near normal laboratory findings may be seen in asymptomatic patients with CLD and cirrhosis. With new and effective treatments becoming available for the treatment of liver disease, identification of such individuals with proper risk factor determination by history, physical examination, and targeted laboratory testing will better insure earlier treatment and potentially decrease the number of patients with both known and unknown advanced and decompensated liver disease.

\section{Disclosure}

The authors report no conflicts of interest in this work.

\section{References}

1. Murphy SL, Xu J, Kochanek KD. Deaths: final data for 2010. Natl Vital Stat Rep. 2013;61(4):1-117.

2. Hoyert DL, Xu J. Deaths: preliminary data for 2011. Natl Vital Stat Rep. 2012;61(6):1-52.

3. NIDDK. Digestive Diseases in the United States: Epidemiology and Impact. Bethesda, MD: NIH Publication; 1994:94-1447.
4. Scaglione SJ, Kliethermes S, Cao G, et al. The epidemiology of cirrhosis in the United States: a population-based study. J Clin Gastroenterol. 2015;49(8):690-696.

5. Li D, Friedman SL. Liver fibrogenesis and the role of hepatic stellate cells: new insights and prospects for therapy. J Gastroenterol Hepatol. 1999;14(7):618-633.

6. Abshagen K, Brensel M, Genz B, et al. Foxf1 SiRNA delivery to hepatic stellate cells by DBTC lipoplex formulations ameliorates fibrosis in livers of bile duct ligated mice. Curr Gene Ther. 2015;15(3):215-227.

7. Han YP. Matrix metalloproteinases, the pros and cons, in liver fibrosis. J Gastroenterol Hepatol. 2006;21(suppl 3):S88-S91.

8. Bataller R, North KE, Brenner DA. Genetic polymorphisms and the progression of liver fibrosis: a critical appraisal. Hepatology. 2003;37(3):493-503.

9. Iredale JP. Models of liver fibrosis: exploring the dynamic nature of inflammation and repair in a solid organ. J Clin Invest. 2007;117(3):539-548.

10. Roy S, Benz F, Luedde T, Roderburg C. The role of miRNAs in the regulation of inflammatory processes during hepatofibrogenesis. Hepatobiliary Surg Nutr. 2015;4(1):24-33.

11. Batts KP, Ludwig J. Chronic hepatitis. An update on terminology and reporting. Am J Surg Pathol. 1995;19(12):1409-1417.

12. Fallowfield J, Hayes P. Pathogenesis and treatment of hepatic fibrosis: is cirrhosis reversible? Clin Med. 2011;11(2):179-183.

13. Tapper EB, Castera L, Afdhal NH. FibroScan (vibration controlled transient elastography): where does it stand in the United States practice. Clin Gastroenterol Hepatol. 2015;13(1):27-36.

14. Wong GL. Transient elastography: kill two birds with one stone? World J Hepatol. 2013;5(5):264-274. 
15. Chon YE, Choi EH, Song KJ, et al. Performance of transient elastography for the staging of liver fibrosis in patients with chronic hepatitis B: a meta-analysis. PLoS One. 2012;7(9):e44930.

16. Chen YP, Liang XE. Noninvasive assessment of liver fibrosis: reduce or substitute the need for liver biopsy? Liver Int. 2015;35(11): 2483.

17. Cabibi D, Calvaruso V, Giuffrida L, et al. Comparison of histochemical staining methods and correlation with transient elastography in acute hepatitis. Pathobiology. 2015;82(1):48-52.
18. Juárez-Hernández E, Uribe-Ramos MH, Ramos-Ostos MH, et al. Factors associated with the quality of transient elastography. Dig Dis Sci. 2015;60(7):2177-2182.

19. Yovchev MI, Xue Y, Shafritz DA, Locker J, Oertel M. Repopulation of the fibrotic/cirrhotic rat liver by transplanted hepatic stem/progenitor cells and mature hepatocytes. Hepatology. 2014;59(1):284-295.

20. Liedtke C, Luedde T, Sauerbruch T, et al. Experimental liver fibrosis research: update on animal models, legal issues and translational aspects. Fibrogenesis Tissue Repair. 2013;6(1):19.
Clinical and Experimental Gastroenterology

\section{Publish your work in this journal}

Clinical and Experimental Gastroenterology is an international, peerreviewed, open access, online journal publishing original research, reports, editorials, reviews and commentaries on all aspects of gastroenterology in the clinic and laboratory. This journal is included on PubMed. The manuscript management system is completely online

\section{Dovepress}

and includes a very quick and fair peer-review system, which is all easy to use. Visit http://www.dovepress.com/testimonials.php to read real quotes from published authors. 